\title{
Orthogonality catastrophe in a one-dimensional system of correlated electrons
}

\author{
V. Meden ${ }^{a}$, P. Schmitteckert ${ }^{b}$, and Nic Shannon ${ }^{c}$ \\ ${ }^{a}$ Department of Physics, Indiana University, Bloomington, Indiana 47405, U. S. A. \\ ${ }^{b}$ IPCMS-GEMME, 23 rue du Loess, 67037 Strasbourg Cedex, France \\ ${ }^{c}$ Department of Physics, University of Warwick, Coventry CV4 7AL, England
}

(July 3, 1997)

\begin{abstract}
We present a detailed numerical study of the orthogonality catastrophe exponent for a onedimensional lattice model of spinless fermions with nearest neighbor interaction using the density matrix remormalization group algorithm. Keeping up to 1200 states per block we achieve a very great accuracy for the overlap which is needed to extract the orthogonality exponent reliably. We discuss the behavior of the exponent for three different kinds of a localized impurity. For comparison we also discuss the non-interacting case. In the weak impurity limit our results for the overlap confirm scaling behavior expected from perturbation theory and renormalization group calculations. In particular we find that a weak backward scattering component of the orthogonality exponent scales to zero for attractive interaction. In the strong impurity limit and for repulsive interaction we demonstrate that the orthogonality exponent cannot be extracted from the overlap for systems with up to 100 sites, due to finite size effects. This is in contradiction to an earlier interpretation given by Qin et al. based on numerical data for much smaller system sizes. Neverthless we find indirect evidence that the backward scattering contribution to the exponent scales to $1 / 16$ based on predictions of boundary conformal field theory.
\end{abstract}

PACS numbers: 71.10.Pm, 72.15Nj, 79.60.-i, 72.15.Qm

\section{INTRODUCTION}

Anderson's "orthogonality catastrophe" is an important and universal piece of metallic physics with far reaching consequences for the response functions of many-electron systems. It is well known that for noninteracting electrons the overlap between the many-body ground states with and without a local impurity potential vanishes in the thermodynamic limit [1]. For a large but finite system of length $L$ the overlap tends to zero as a power law $L^{-\alpha}$, defining an orthogonality exponent $(\mathrm{OE}) \alpha$. This orthogonality catastrophe is closely related to the $\mathrm{x}$-ray edge problem in metals [2,3], and in this context the exponent $\alpha$ can often be measured directly.

As was first observed by Anderson [1] the OE for a spherically symmetric impurity in a free electron gas (in arbitrary dimension) is uniquely determined by the square of the scattering phase shifts of the infinite system at the Fermi energy $E_{F}$. The proof of this result for more general potentials is far from trivial $[2,4,5]$. For spinless one-dimensional (1D) Fermions we can write

$$
\alpha=\frac{1}{2}\left(\frac{\delta_{e}\left(E_{F}\right)}{\pi}\right)^{2}+\frac{1}{2}\left(\frac{\delta_{o}\left(E_{F}\right)}{\pi}\right)^{2},
$$

with the phase shifts of the even and odd scattering channels. The remaining problem is the calculation of the phase shifts for a given impurity potential. In the following $\delta$ always denotes the phase shift at the Fermi energy.

In one dimension the phase shifts can generally be expressed in terms of the reflection and transmission coefficients of an incident wave packet [6]

$$
\delta_{e / o}=\frac{1}{2}\left[\varphi_{T} \mp \arcsin \{|R|\}\right],
$$

with the phase $\varphi_{T}$ of the transmission coefficient and the absolute value $|R|$ of the reflection coefficient taken at the Fermi energy. If we introduce new phase shifts $\delta_{f / b}$ given by

$$
\delta_{e / o}=\frac{1}{2}\left(\delta_{f} \mp \delta_{b}\right),
$$

we can identify the forward scattering phase shift $\delta_{f}$ with $\varphi_{T}$ and the backward scattering phase shift $\delta_{b}$ with $\arcsin \{|R|\}$. Expressed in terms of $\delta_{f / b}$ or $|R|$ and $\varphi_{T}$, the $\mathrm{OE}$ is given by

$$
\begin{aligned}
\alpha & =\alpha_{f}+\alpha_{b} \\
& =\frac{1}{4}\left(\frac{\delta_{f}}{\pi}\right)^{2}+\frac{1}{4}\left(\frac{\delta_{b}}{\pi}\right)^{2} \\
& =\frac{1}{4 \pi^{2}}\left[\varphi_{T}^{2}+\arcsin ^{2}\{|R|\}\right],
\end{aligned}
$$

defining the forward $\left(\alpha_{f}\right)$ and backward $\left(\alpha_{b}\right)$ scattering contributions to the OE.

In the lowest order perturbation theory in the impurity strength (the Born approximation) it is always possible to express the scattering phase shifts $\delta_{f / b}$ in terms of the matrix elements of the impurity term $\hat{W}$ in the Hamiltonian. If we denote the one-particle eigenstates of the impurity free Hamiltonian with energy $E_{F}$ by $\left| \pm k_{F}\right\rangle$, we obtain

$$
\begin{aligned}
& \left(\delta_{f}^{B}\right)^{2}=\frac{1}{v_{F}^{2}}\left|\left\langle k_{F}|\hat{W}| k_{F}\right\rangle\right|^{2} \\
& \left(\delta_{b}^{B}\right)^{2}=\frac{1}{v_{F}^{2}}\left|\left\langle-k_{F}|\hat{W}| k_{F}\right\rangle\right|^{2} .
\end{aligned}
$$

Correlation effects are very important in one dimension, where it is known that even an infinitesimally small 
interaction between electrons changes their low energy properties from those of a Fermi to those of a Luttinger liquid (LL) [7]. The low energy excitations of a LL are collective and bosonic, so the concept of phase shifts in fermionic one-(quasi-)particle wave functions is clearly no longer applicable, contrary to the situation in interacting Fermi liquids where this is still possible. It is therefore interesting to ask whether the orthogonality catastrophe in a $1 \mathrm{D}$ system is also dramatically altered by electronelectron interaction.

In this article we analyze the problem by numerically calculating the overlap

$$
O \equiv\left|\left\langle E_{0} \mid E_{0}^{I}\right\rangle\right|
$$

and the difference in the ground state energies

$$
\Delta E \equiv\left|E_{0}^{I}-E_{0}\right|
$$

between the ground states of the system with $\left(\left|E_{0}^{I}\right\rangle\right)$ and without $\left(\left|E_{0}\right\rangle\right)$ the impurity for three different kinds of impurity in a lattice model of spinless interacting Fermions. We distinguish between the weak and strong impurity limit. The results are compared with the predictions of perturbation theory and the application of renormalization group ( $\mathrm{RG}$ ) ideas. Large system sizes and very accurate calculations are found to be necessary to reliably determine $\alpha$. These can be achieved by using the density matrix renormalization group algorithm (DMRG) $[8,9]$. This approach makes it possible to calculate the ground state properties of an interacting system with an accuracy comparable to that of exact diagonalization, but for much larger system sizes. Using this technique, we are able to work with chains of up to 100 sites, keeping up to 1200 states per block and performing 7 finite lattice sweeps in the DMRG procedure.

The behavior of $1 \mathrm{D}$ correlated electrons in the presence of a localized impurity is of general interest because the physics of the problem manifests itself in a number of different contexts, e. g. the conductivity of quasi 1D "metallic" materials [10], tunneling through a constriction in the fractional quantum Hall regime [11] and Friedel oscillations in the charge density of highly anisotropic systems [12-14]. Given the rapid progress in the fabrication of quasi 1D metallic systems (highly anisotropic materials or quantum wires) it should in the future be possible to measure the $\mathrm{x}$-ray response of these systems directly, and so fit $\alpha$ to experiments.

Considerable progress has been made in the understanding of the interplay between impurity and interaction in one dimension since the seminal work of Kane and Fisher [10]. Kane and Fisher discussed the problem of a single impurity in a 1D wire within the effective low energy field theory of 1D correlated electrons, using a perturbative RG approach. If one integrates out high momentum degrees of freedom any weak $2 k_{F}$ component of the impurity potential increases for repulsive interaction and scales to zero for attractive ones. Thus backward scattering of electrons is relevant for repulsive interaction and irrelevant for attractive interaction. Kane and Fisher also discussed the dual problem of a weak hopping between the two ends of an open chain. The strength of this weak link flows to zero for repulsive interaction, but is relevant for attractive interaction.

It is tempting to conclude that, for repulsive interaction, the (weak) $2 k_{F}$ component of the impurity potential scales to infinity and therefore the open chain fixed point (open chain interpretation). However it is not immediately obvious that a single impurity in a LL really does correspond to a "cut wire".

In order to shed more light on this issue Kane and Fisher calculated the conductivity of a $1 \mathrm{D}$ wire with a localized impurity exactly for a special value of the LL interaction parameter. Their result is consistent with this interpretation of the RG results [10]. Later authors calculated the conductivity for arbitrary interaction strength $[15,16]$, again obtaining results consistent with the open chain interpretation. Further supporting evidence for this picture was given by a numerical calculation of the conductivity [11]. Similar arguments hold in the dual situation of (strong) hopping between two semi-infinite LL's, attractive interaction, and the periodic chain fixed point (periodic chain interpretation).

However the question of how the RG results should be interpreted in the context of the OE remains controversial $[17-22]$.

A number of attempts have been made to calculate the orthogonality exponent (or equivalently the $\mathrm{x}$-ray edge exponent) of a 1D interacting electron gas with strong impurities in the light of Kane and Fisher's RG results [17-19]. All of these approaches are based on the Tomonaga-Luttinger (TL) Hamiltonian, the effective low energy continuum field theory for most models of $1 \mathrm{D}$ correlated electrons in the absence of impurities. The TL Hamiltonian is written in terms of distinct left and right moving fermion fields $\psi_{ \pm}(x)$. The low energy physics of a given microscopic model can be mapped onto a TL model by determining the LL parameter $K$ and the renormalized velocity $v$ of the model in question, in terms of its microscopic parameters [23]. Impurity scattering is given by two types of term in the Hamiltonian. Forward scattering can be written linearly in the density of right and left moving Fermions. If only this kind of scattering is present then the calculation of the overlap within the field theoretical model is straightforward (see Section II C). Backward scattering couples right and left moving fields and results in a complicated sine-Gordon like term in the field theory [10].

To calculate the overlap within the field theory in the presence of backward scattering further approximations are needed. To solve the problem for repulsive electronelectron interaction Gogolin [17] and Prokof'ev [18] used a strong backward scattering approximation, replacing the cosine of the boson field by a term quadratic in the field. This replacement is consistent with the open chain interpretation of the $\mathrm{RG}$ results and leads to a backward 
scattering contribution

$$
\alpha_{b}=\frac{1}{16}
$$

to the OE, which is completely independent of the strength of the interaction or the size of the bare backward scattering potential.

This result is frequently interpreted in terms of phase shifts. According to the open chain interpretation of Kane and Fisher's scaling analysis outlined above, the $2 k_{F}$ component of the impurity is renormalized to infinity. This leads to perfect reflection (reflection coefficient $R=1$ ) and a backward scattering phase shift of $\pi / 2$. Introducing this phase shift into Eq. (4) gives Eq. (9). As there are no fermionic single-particle scattering wave functions which are eigenfunctions of the interacting Hamiltonian, this interpretation only has a meaning if one extends the concept of phase shifts. We can do this by regarding the phase shift at the Fermi energy as the number determining the boundary condition between in- and outgoing field operators according to $\psi_{\text {out }}=\exp (2 i \delta) \psi_{\text {in }}$. In this sense the above interpretation does still have a physical meaning [24].

Beside the direct calculation of the overlap, an alternative method of calculating the $\mathrm{OE}$ is given by boundary conformal field theory (BCFT) $[24,25]$. BCFT relates the properties of the boundary changing operator associated with the introduction of a single impurity in a (semi-) infinite 1D interacting Fermi system to the finite size spectrum of an equivalent field theory on a line of length $L$. For non-interacting electrons and for interacting electrons with only a forward scattering impurity component the difference $\Delta E$ of the ground state energies of the finite size system with and without the impurity can be calculated directly from a mode expansion in the effective low energy field theory on the line. In the absence of bound state effects $\Delta E$ is given by

$$
\lim _{L \rightarrow \infty} L \Delta E=\lim _{L \rightarrow \infty} L \Delta e+2 \pi v \alpha
$$

where $\Delta e$ is a bulk constant and $v$ the renormalized charge velocity of the field. For interacting electrons this result enables us to calculate $\alpha_{f}$ directly from numerically determined ground state energies [24,26].

An alternative derivation of Eq. (1) for non-interacting electrons based on Eq. (10) has been given [25]. This approach has also been used in conjunction with the Bethe Ansatz to obtain predictions for the orthogonality exponent in lattice systems with integrable defects [27]. Additionally it is possible to calculate the ground state energy difference $\Delta E$ in the presence of an infinitely strong backward scattering potential. If one assumes that the scaling of the $2 k_{F}$ component of the potential for repulsive electron-electron interaction finally leads to an open boundary condition fixed point one again obtains $\alpha_{b}=1 / 16$.

It is important to notice that all the theoretical results discussed so far are based on, or are equivalent to, the open chain interpretation of the results of the perturbative RG.

Recently the overlap $O$ Eq. (7) has been discussed within the low energy continuum field theory for the special LL parameter $K=1 / 2$ corresponding to a strong repulsive interaction $[28,29]$. At this point it is possible to calculate the overlap by bosonization and refermionization. The result is again consistent with $\alpha_{b}=1 / 16$.

In Refs. [20] and [21] Oreg and Finkel'stein discuss the tunneling density of states near a localized impurity and the $\mathrm{x}$-ray edge exponent based on the mapping of the TL model onto a Coulomb gas model. They obtain results in contradiction with results obtained by all other authors. Whether their calculations suffer from a flaw in the anticommutation relations of the Fermion field operators is still a matter of contention [22,28-31].

In Refs. [30] and [31] Qin et al. use the DMRG method to calculate the overlap for a very special type of localized impurity. We will later comment on the relation between their results and ours.

The remainder of this article is organized as follows: In Sec. II we introduce the model considered, discuss analytical results for the $\mathrm{OE}$ in the non-interacting case, and derive the results for the $\mathrm{OE}$ in lowest order perturbation theory in the impurity strength, based on the effective low energy field theory for interacting electrons. In order to obtain the $\mathrm{OE}$ it is necessary to extrapolate finite size data for the overlap and the energy difference to the thermodynamic limit. This leads to an extrapolation error. An additional error is introduced because the DMRG gives only an approximate result for the ground state properties. We discuss both errors carefully in Sec. III by calculating the overlap $O$ and $\Delta E$ numerically for the non-interacting model and comparing with the exact results derived in Sec. II B.

For non-interacting electrons a straightforward numerical calculation of $O$ and $\Delta E$ is possible for very large system sizes, if we take the Slater determinant structure of the ground states into account. The comparison of exact results for non-interacting electrons makes it possible to access the errors involved in the DMRG procedure. It turns out that this kind of error analysis is crucial for the interpretation of our results. In Sec. IV we present our numerical results and analyze the data with respect to the expected scaling behavior. Finally we summarize our results in Sec. V. In the Appendix we briefly describe how to obtain perturbative results in the weak impurity and weak hopping limits.

\section{MODELS AND ANALYTICAL RESULTS}

\section{A. The Hamiltonian}

In our investigation we consider a model of $N$ spinless Fermions on a 1D lattice of $M$ sites with site dependent 
on-site energies $\varepsilon_{i}$, nearest neighbor hopping $t$ and interaction $V$, described by the Hamiltonian

$$
\begin{aligned}
H= & -t \sum_{i=1}^{M}\left(c_{i}^{\dagger} c_{i+1}+c_{i+1}^{\dagger} c_{i}\right)+\sum_{i=1}^{M} \varepsilon_{i} c_{i}^{\dagger} c_{i} \\
& +V \sum_{i=1}^{M} n_{i} n_{i+1}+(b-1)\left[-t\left(c_{M}^{\dagger} c_{1}+c_{1}^{\dagger} c_{M}\right)\right] \\
& +\left(b^{\prime}-1\right)\left[V n_{M} n_{1}\right]
\end{aligned}
$$

where $c_{i}^{(\dagger)}$ denotes the Fermion lowering (raising) operator at the site $i$, and $n_{i}$ the related density operator. In all sums we identify $M+1 \equiv 1$. By choosing $b=b^{\prime}=1$ and $\varepsilon_{i}=0$ for all $i$ we obtain an impurity free Hamiltonian for interacting Fermions with periodic boundary conditions (PBC). It is well known that this Hamiltonian displays LL behavior, and for the case of a half filled band the LL parameter $K$ and the renormalized velocity $v$ can be obtained from the finite size corrections [32] to the Bethe Ansatz [33] solution

$$
\begin{aligned}
v & =t \frac{\pi \sin (2 \eta)}{\pi-2 \eta}, \\
K & =\frac{\pi}{4 \eta}
\end{aligned}
$$

where $\eta$ parameterizes the interaction

$$
V=-2 t \cos (2 \eta)
$$

For $V>0$ (repulsive interaction), $K<1$, and for $V<0$ (attractive interaction) $K>1$. In this article we will focus on the half filled case. According to the LL theory of 1D correlated electrons [7] $K$ and $v$ completely determine the low-energy behavior of the Hamiltonian. It is known that in the absence of impurities the model described by Eq. (11) undergoes a transition into a charge density wave ground state at $V /|t|=2$, and into a phase separated state at $V /|t|=-2$. We are therefore limited to parameters $V /|t|$ within $(-2,2)$ leading to $K \in(1 / 2, \infty)$.

By varying the parameters $\varepsilon_{i}, b$ and $b^{\prime}$ we can introduce different kinds of localized impurity to the Hamiltonian Eq. (11). In this article we consider three kinds of impurity. Setting $\varepsilon_{1}=\varepsilon, \varepsilon_{i}=0$ for $i \neq 1$ and $b=b^{\prime}=1$ creates a site impurity on one of the lattice sites. Without loss of generality we have chosen the site 1 as the impurity site. Physically one might think of this kind of term as an impurity ion sitting on one of the sites of the regular lattice, or as the core hole generated by removing one (or more) of the core electrons of the atom at site 1 ( $\mathrm{x}$-ray edge problem). In this article we will focus on repulsive impurities $(\varepsilon>0)$ to avoid complications due to bound states. A hopping impurity is obtained by choosing $\varepsilon_{i}=0$ for all $i, b^{\prime}=1$ and $0 \leq b<1$ and might be created by an interstitial between lattice sites $M$ and 1 which changes the hopping amplitude but not the interaction between them.
Qin et al. [30,31] recently discussed a third type of impurity. These authors worked with the Heisenberg XXZ spin $1 / 2$ chain, which can be mapped onto a model of spinless Fermions by a Wigner-Jordan transformation [34]. In terms of the Heisenberg model, their impurity is generated by weakening the coupling of the $x-, y$ - and $z$-components of the spin between sites $M$ and 1 by an equal amount. In the Fermion basis, this impurity corresponds to an exotic combination of modified hopping and interaction between the sites $M$ and 1, accompanied by site impurities at the sites $M$ and 1 . Their model is equivalent to Eq. (11) with $\varepsilon_{1}=\varepsilon_{M}=-(b-1) V / 2, \varepsilon_{i}=0$ for all the other $i, b=b^{\prime}$ and $0 \leq b<1$. For comparison we will also discuss this type of impurity, and denote it a bond impurity. It is equivalent to the hopping impurity in the non-interacting limit $V=0$.

The ground state of the Hamiltonian Eq. (11) without impurities is degenerate for even particle number $N$. There are several ways of overcoming the problems associated with this degeneracy and (physical) even-odd effects. One straightforward solution is to calculate $O$ and $\Delta E$ only for odd $N$. However, in order to have access to more data points we have chosen a different approach in the majority of our numerical calculations. For even $N$ we altered the hopping between the sites $M$ and 1 from $t$ to $-t$. This lifts the degeneracy of the ground state, and prevents even-odd effects irrelevant to the orthogonality catastrophe considered in this article. One can implement this in the Hamiltonian by the direct substitution

$$
(b-1) \longrightarrow\left(-\exp \left\{i \pi \sum_{j=1}^{M} c_{j}^{\dagger} c_{j}\right\} b-1\right)
$$

in the second line of Eq. (11). If one works with the Heisenberg Hamiltonian and imposes PBC's for the spins this additional phase factor emerges as a natural consequence of the Wigner-Jordan transformation [34].

\section{B. The orthogonality exponent for non-interacting electrons}

As shown by Eq. (4), we can determine the $\mathrm{OE} \alpha$ for non-interacting electrons by solving the one-particle scattering problem and calculating $|R|$ and $\varphi_{T}$. For the types of impurities considered in this article this is straightforward and can be done following e. g. Ref. [35]. For the site impurity we obtain

$$
\alpha=\frac{1}{2 \pi^{2}} \arcsin ^{2}\left\{\frac{\varepsilon}{\sqrt{4 t^{2}+\varepsilon^{2}-E_{F}^{2}}}\right\} .
$$

The forward and backward scattering contributions $\alpha_{f / b}$ are both given by one-half of $\alpha$, as $\varphi_{T}=-\arcsin |R|$, and $|R|$ is given by the argument of the arcsine in Eq. (16). In preparation for the discussion of the OE in terms of an effective low energy field theory in the next section, 
we also calculate $\delta_{f / b}$ in the Born approximation and at half filling. This will be necessary in order to relate the impurity parameters of the microscopic lattice model to the impurity potentials in the field theory. We denote the value of the $\mathrm{OE}$ obtained from the Born approximation as $\alpha^{B}$. Following Eqs. (5) and (6) we obtain $\left(\delta_{f}^{B}\right)^{2}=$ $\left(\delta_{b}^{B}\right)^{2}=\left(\varepsilon / v_{F}\right)^{2}$ with the non-interacting Fermi velocity $v_{F}=2|t|$. Inserting this result into Eq. (4) leads to the same result as an expansion of Eq. (16) to lowest order in $\varepsilon$

$$
\alpha^{B}=\frac{1}{2 \pi^{2}}\left(\frac{\varepsilon}{v_{F}}\right)^{2}
$$

The next order corrections to $\alpha^{B}$ are of the order $\left(\varepsilon / v_{F}\right)^{4}$.

To simplify the calculation in the case of the hopping impurity we concentrate on the half filled band with $E_{F}=0$. In this case

$$
\alpha=\frac{1}{4 \pi^{2}} \arcsin ^{2}\left\{\frac{\beta-\beta^{2} / 2}{1-\beta+\beta^{2} / 2}\right\},
$$

with $\beta=(1-b)$. For the hopping impurity at half filling the forward scattering contribution vanishes, e. g. we obtain

$$
\delta_{f} \equiv \varphi_{T}=0
$$

and $|R|$ is again given by the argument of the arcsine in Eq. (18). For a weak impurity (small $\beta$ ) an expansion leads to

$$
\alpha^{B}=\beta^{2} /\left(4 \pi^{2}\right)
$$

and $\left(\delta_{b}^{B}\right)^{2}=\beta^{2}$. In contrast to the site impurity the next order corrections to the Born approximation are of the order $\beta^{3}$ and therefore more important than for the site impurity. This observation will be important in what follows. As the impurity strength in the lattice model is not determined by a local impurity potential, the calculation of the backward scattering phase shift in the Born approximation following Eq. (6) is a little bit more involved, but also leads to Eq. (20).

\section{Bosonization and perturbation theory in the impurity strength}

In the low energy, long wavelength limit, the impurity free Hamiltonian can be written in terms of a bosonized continuum field theory [23]. The new effective Hamiltonian is given by

$\mathcal{H}_{0}=\frac{v}{2 \pi} \int_{-L / 2}^{L / 2} d x\left\{K \pi^{2} \Pi^{2}(x)+\frac{1}{K}\left(\frac{\partial \Phi(x)}{\partial x}\right)^{2}\right\}$,

and the canonical conjugate bosonic fields $\Phi(x)$ and $\Pi(x)$ can be expressed in terms of the densities $\rho_{ \pm}$of right and left moving Fermions [23]

$$
\begin{aligned}
\Pi(x)= & \rho_{+}(x)-\rho_{-}(x), \\
\Phi(x)= & -i \frac{\pi}{L} \sum_{q \neq 0} \frac{1}{q} e^{-i q x}\left[\rho_{+}(q)+\rho_{-}(q)\right] \\
& -\frac{\pi x}{L}\left[\hat{N}_{+}+\hat{N}_{-}\right],
\end{aligned}
$$

where the number operators $\hat{N}_{ \pm}$measure the particle number with respect to a fixed ground state. In the noninteracting model $K=1$ and the velocity $v$ is given by the non-interacting Fermi velocity $v_{F}$.

A forward scattering impurity can be expressed as

$$
\mathcal{W}_{f}=\int_{-L / 2}^{L / 2} W_{f}(x) \rho(x),
$$

with the total density $\rho(x)=\left[\rho_{+}(x)+\rho_{-}(x)\right]$ and the local potential $W_{f}(x)$. Eq. (23) implies

$$
\rho(x)=-\frac{1}{\pi} \frac{\partial \Phi(x)}{\partial x} .
$$

Since $\mathcal{W}_{f}$ is linear in the $\rho_{ \pm}(q)$, the ground states of $\mathcal{H}_{0}$ and $\mathcal{H}_{0}+\mathcal{W}_{f}$ are related by a unitary transformation and the overlap can be calculated explicitly

$$
O_{f}=\exp \left\{-\frac{1}{2 \pi L} \frac{K}{v^{2}} \sum_{q>0} \frac{\left|\tilde{W}_{f}(q)\right|^{2}}{q}\right\} .
$$

Taking the limit $L \rightarrow \infty$ we can determine the OE $[12,36]$

$$
\alpha_{f}=\frac{K}{4 \pi^{2}} \frac{\left|\tilde{W}_{f}(0)\right|^{2}}{v^{2}} .
$$

Anderson's orthogonality catastrophe is therefore still present in an interacting system with forward scattering, but the OE is modified. Eq. (27) trivially reproduces the non-interacting limit, and we conclude from comparison with Eq. (5) that the field theoretical description always gives the Born approximation in $W_{f}$ for the $\mathrm{OE}$ [37]. We therefore anticipate that for $V \neq 0$, Eq. (27) also holds only for weak forward scattering.

This raises the general question of how the specific impurities (e. g. bond, site, hopping) of a given microscopic model should be mapped onto the impurity potential $\tilde{W}_{f}(q)$ of the effective field theory. Comparing Eq. (27) for $V=0$ with Eq. (4) we find

$$
\frac{\left|\tilde{W}_{f}(0)\right|^{2}}{v_{F}^{2}}=\delta_{f}^{2}
$$

where $\delta_{f}$ is the exact non-interacting forward scattering phase shift of the underlying microscopic model. Within this mapping we obtain from the field theoretical Born approximation the exact result for $\alpha_{f}$ of the microscopic model. But it is not clear whether this identification also gives a systematic improvement in the interacting 
case. From a systematic point of view we can only identify the impurity parameters in lowest order in perturbation theory $\left[\tilde{W}_{f}(0) / v_{F}\right]^{2}=\left(\delta_{f}^{B}\right)^{2}$, where $\delta_{f}^{B}$ is the noninteracting microscopic forward scattering phase shift in the Born approximation.

For small $\left|\tilde{W}_{f}(0)\right|$ we can therefore write

$$
\alpha_{f}^{B}(V)=K \frac{v_{F}^{2}}{v^{2}} \alpha_{f}^{B}(V=0)
$$

Having calculated $\alpha_{f}^{B}(V=0)$ for our microscopic site impurity in Eq. (17), $\alpha_{f}^{B}(V)$ is determined by Eq. (29). We will later compare the values of $\alpha_{f}$ predicted by this equation with our numerical results for weak site impurities.

To the best of our knowledge there are no analytical results for the case of strong forward scattering and $V \neq 0$.

It is important to note that the inclusion of the forward scattering term Eq. (24) in the Hamiltonian breaks its particle-hole symmetry. As the lattice Hamiltonians Eq. (11) for the hopping and bond impurities at half filling are particle-hole symmetric, in this special case the mapping of the microscopic model onto an effective field theory cannot give rise to such a term. In this sense the forward scattering contribution to the $\mathrm{OE}$ vanishes for interacting electrons as well, which simplifies the interpretation of some of our results.

For purposes of comparison with numerical data for finite size systems, it is interesting to evaluate Eq. (26) with finite $L$. Provided that we can expand $\left|\tilde{W}_{f}(q)\right|^{2}$ in a power series in $q$, the Euler summation formula [38] gives

$O=\exp \left\{-\alpha_{f} \ln (L)+a_{0}+a_{1} L^{-1}+a_{2} L^{-2}+\ldots\right\}$,

with impurity strength and interaction dependent constants $a_{i}$. In the Appendix we will argue that in the presence of backward scattering the finite size corrections for $V=0$ are also given by integer powers of $1 / L$.

The backward scattering contribution to the low energy field theory is given by

$$
\mathcal{W}_{b}=\int_{-L / 2}^{L / 2} W_{b}(x)\left[\psi_{+}^{\dagger}(x) \psi_{-}(x)+\text { h.c. }\right],
$$

where the field operators $\psi_{ \pm}(x)$ can be expressed in terms of the Boson fields $\Pi(x)$ and $\Phi(x)[7,23]$. In the Appendix we derive an expression for the overlap perturbatively in the backward scattering potential. If only backward scattering is present [12]

$$
O_{b}=1-a_{1}(V) \frac{W_{b}^{2}}{v_{F}^{2}}-a_{2}(V) \frac{W_{b}^{2}}{v_{F}^{2}} L^{2-2 K}+\ldots,
$$

where the $a_{i}(V)$ are interaction dependent constants, $W_{b}$ is a measure of the backward scattering strength and the dots denote terms of second order in $W_{b} / v_{F}$ falling off as $1 / L$ or faster and terms of higher order in $W_{b} / v_{F}$. This result is consistent with the RG equations given in Ref.
[10], and is related to the anomalous time dependence of the core-hole Green's function discussed in Ref. [17]. It is not obvious from Eq. (32) that we will recover the non-interacting form

$$
O_{b}=1-a_{1} \frac{W_{b}^{2}}{v_{F}^{2}}-\alpha_{b}^{B} \ln (L)+\ldots
$$

in the limit $K \rightarrow 1$. By taking the explicit $V$ dependence of the coefficients $a_{i}(V)$ into account, we show in the Appendix that this is in fact the case.

For repulsive interaction $(K<1)$, the expansion Eq. (32) breaks down at large system sizes, even if the bare backward scattering potential is weak. The same is true in the non-interacting case, but the logarithmic divergence there is replaced by a faster power law divergence. As we do not find the usual behavior Eq. (33) in the interacting case, we expect that the orthogonality catastrophe in the presence of backward scattering and interaction is drastically altered. For attractive interaction $(K>1)$ and in the lowest order perturbation theory $O_{b}$ tends to a constant. Provided that this holds also in higher orders, there is no orthogonality catastrophe, i. e. the two ground states are not orthogonal to each other. Where the bare $\left|W_{b}\right|$ is small, we expect to find the scaling behavior Eq. (32) in our numerical data for the overlap, for intermediate system sizes. Terms of higher order in $1 / L$ will be important for very small systems.

As in the forward scattering case a systematic mapping between the microscopic backward scattering parameter and $W_{b}$ is only possible within perturbation theory. In lowest order we can identify $\left[W_{b}(0) / v_{F}\right]^{2}=\left(\delta_{b}^{B}\right)^{2}$, where $\delta_{b}^{B}$ is the non-interacting microscopic backward scattering phase shift in the Born approximation.

In Ref. [10] Kane and Fisher discuss the strong impurity limit by introducing a weak hopping in an open chain and mapping this problem to a field theory dual to Eq. (21). This mapping makes it possible to treat the problem perturbatively in the weak hopping. In our case this corresponds to a calculation of the overlap between the state with the weak hopping (the large hopping or bond impurity) and the chain with open boundary conditions (OBC). To distinguish this overlap from the one discussed previously, we denote it $O^{\circ}$, and the related $\mathrm{OE}$ $\alpha^{o}$. As discussed in the Appendix we can calculate $O_{b}^{o}$ perturbatively in the weak hopping limit in a similar way as $O_{b}$ and the result is given by an expression equivalent to Eq. (32) but with $K$ replaced by $1 / K$

$$
O_{b}^{o}=1-\tilde{a}_{1}(V) \frac{t_{b}^{2}}{t^{2}}-\tilde{a}_{2}(V) \frac{t_{b}^{2}}{t^{2}} L^{2-2 / K}+\ldots
$$

where $t_{b}$ is now a measure of the weak hopping. The roles of repulsive and attractive interaction are interchanged and $O_{b}^{o}$ tends to a constant for repulsive interaction. We will verify Eq. (34) numerically in Sec. IV B.

BCFT predicts that $\alpha_{b}$ between the OBC and the PBC ground states is $1 / 16$, and that this result is independent of $V$ [24]. Bearing in mind all the results discussed so far, 
it is therefore tempting to conclude that $\alpha_{b}(V<0)=0$ and $\alpha_{b}(V>0)=1 / 16$ independent of the bare impurity strength. We want to emphasize that these generalizations of the above perturbative results are consistent with the periodic and open chain interpretation of the perturbative RG results, but need to be verified. For our models we will discuss this question in Sec. IV.

In the next section we will discuss the two major sources for errors in our numerical calculation of the OE: The extrapolation error, and the error due to the approximative nature of the DMRG. In this section we will also introduce the method used to analyze the numerical data for $O$ and $\Delta E$ in order to obtain $\alpha$.

\section{ACCURACY OF THE NUMERICAL RESULTS}

\section{A. Extrapolation to infinite system size}

For non-interacting electrons, the ground states with and without the impurity are Slater determinants of single electron states, and the overlap can be written as the determinant of a matrix whose elements are given by the overlaps between these single electron wave functions [1]. The single particle Hamiltonian can be diagonalized numerically, and it is therefore possible to calculate $O$ for quite large system sizes with an error limited only by machine accuracy. To discuss the finite size behavior of the overlap and hence the $\mathrm{OE}$, we calculated $O$ and $\Delta E$ for non-interacting systems with $M$ up to 1200 .

We expect $O$ to be given by Eq. (30) with $\alpha_{f}$ replaced by $\alpha$. We can determine $\alpha$ by calculating $O$ for different system sizes $M[i], i=1,2,3, \ldots$, taking the centered differences

$$
\alpha^{O}[i]=-\frac{\ln \{O(M[i+1])\}-\ln \{O(M[i-1])\}}{\ln \{M[i+1]\}-\ln \{M[i-1]\}}
$$

and extrapolating by fitting a power law $a+b x+c x^{2}$ with $x=1 / M$ in some window of small $1 / M$.

Alternatively we can calculate $\alpha$ using the results of BCFT Eq. (10). For non-interacting electrons BCFT predicts that the finite size corrections are given by integer powers of $1 / M[24,25]$. Thus we calculate $\Delta E$ for different $M[i]$, take the centered differences

$$
\alpha^{\Delta E}[i]=\frac{1}{2 \pi v}\left|\frac{\Delta E(M[i+1])-\Delta E(M[i-1])}{(1 / M[i+1])-(1 / M[i-1])}\right|
$$

and once again fit the data with a quadratic polynomial in $1 / M$.

Fig. 1 shows $\alpha^{O}[i]$ and $\alpha^{\Delta E}[i]$ for the site and hopping impurities in the strong impurity limit with $\varepsilon /|t|=3$ and $b=0.1$ at half filling. The results discussed in this section are quite general and not limited to the case of strong impurities. From Fig. 1 it is obvious that we can confirm the prediction of BCFT Eq. (10). The exact values of

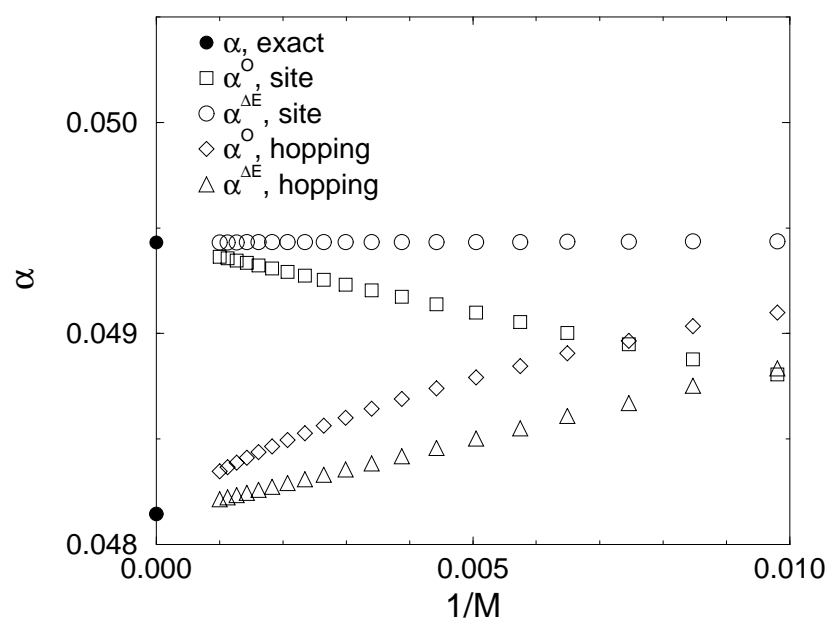

FIG. 1. The non-interacting orthogonality exponent extrapolated from a finite size scaling of the overlap and the energy difference for a site and a hopping impurity with $\varepsilon /|t|=3$ and $b=0.1$.

$\alpha$ obtained from Eqs. (16) and (18), and the absolute extrapolation errors of a finite size scaling in different $1 / M$ windows are given in Table I. Fitting only the data with $M[i] \in[200,1200]$ we obtain numerical values for $\alpha$ with a very high accuracy. For $M[i] \in[50,200]$ which is comparable to the range accessible by the DMRG the relative extrapolation error is between $10^{-2}$ and $10^{-5}$, depending on the impurity type and whether the extrapolation is of $\alpha^{O}[i]$ or $\alpha^{\Delta E}[i]$. Generally the accuracy is at least one order of magnitude better for the site impurity than it is for the hopping impurity. We therefore conclude that while the hopping impurity has the advantage of generating only backward scattering, its finite size corrections are much more important than those for the site impurity. As there is no reason to believe that the extrapolation error for interacting electrons is smaller than that for non-interacting electrons this observation will be important in what follows. We also notice that the value of $\alpha$ obtained by the extrapolation of $\alpha^{\Delta E}[i]$ is at least one order of magnitude better than the one from $\alpha^{O}[i]$.

\section{B. Accuracy of the DMRG}

The DMRG algorithm is a real space blocking method that works particularly well for the calculation of the ground state energies and wave functions of $1 \mathrm{D}$ electronic lattice models. The accuracy of the method is principally limited by the number $m$ of states that are kept after the diagonalization of two joint blocks. A compromise must be reached between keeping $m$ small in order to reduce the numerical effort, and retaining enough states to give a good description of the system built out of the blocks. 


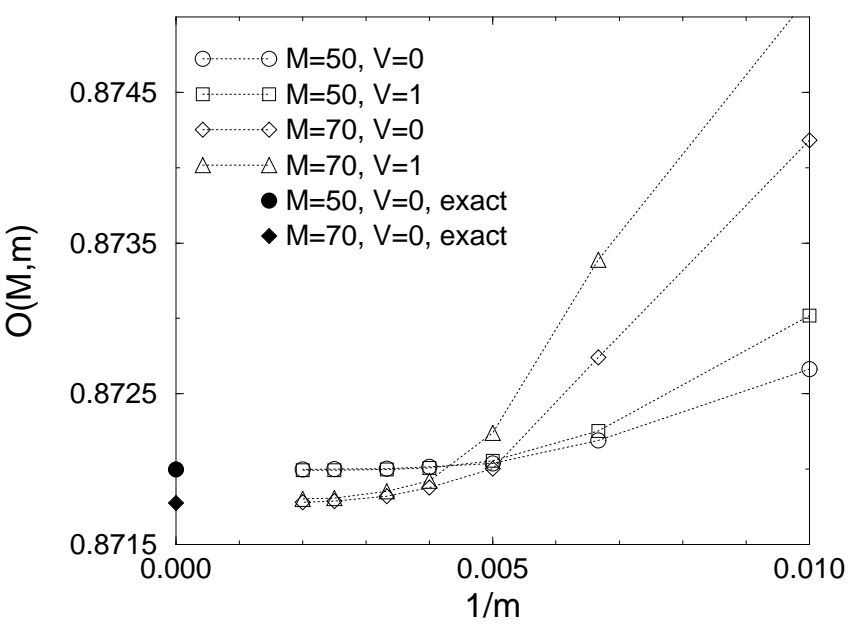

FIG. 2. The overlap as a function of $1 / m$. The full symbols are the exact result for $V=0$ obtained by diagonalizing the one-particle Hamiltonian. For comparison, the full diamond is shifted by 0.014 . The open symbols are the DMRG results. The circles are the overlap for $M=50, V=0$ and a hopping impurity with $b=0.1$. The squares are the data for $M=50$, $V /|t|=1$ and a bond impurity with $b=0.1$. The data are shifted by 0.03026 . The diamonds and triangles are the data for the same parameters but $M=70$. The diamonds are shifted by 0.014 and the triangles by 0.0474 . In the legend $V$ is measured in units of $|t|$.

As the non-interacting case is not a special situation for DMRG, a comparison with the "exact" results for $O$ calculated as in Sec. III A gives us a reliable measure of its error. We therefore used the DMRG to numerically calculate $O(M, m)$ for the site and hopping impurity with different impurity parameters, different $M$ and $m$. For the same impurity parameter and $M$ we calculated $O(M, \infty)$ as in Sec. III A. As a generic example we show in Fig. $2 O(M, m)$ for a hopping impurity with $V=0$ and $b=0.1$ as a function of $1 / m$ for $M=50$ and $M=70$. Additionally the figure shows $O(M, m)$ for the bond impurity with $V /|t|=1$ and $b=0.1$. These are the parameters used in Ref. [30]. To present the curves for the different $M$ and $V$ in one figure the data for $V=0$, $M=70$ and $V /|t|=1, M=50,70$ are shifted as indicated in the caption. It is very important to notice that for all $M$ the overlap approaches the $m=\infty$ limit from above and that $O(M, m)-O(M, \infty)$ is increasing very fast with $M$. To calculate $\alpha$ from $O(M, m)$ we have to take a numerical derivative (see Eq. (35)). If we fix $m$ and increase $M, O(M, m)$ is too large and $O(M, m)-O(M, \infty)$ increases exponentially with $M$. The numerical differentiation in Eq. (35) reinforces this effect and we recover values of $\alpha[i]$ which are systematically too small. For large $M[i]$ this leads to a significant error in $\alpha[i]$.

Although we do not know $O(M, \infty)$ for $V \neq 0$ it is obvious from Fig. 2 that the behavior of $O(M, m)$ is quite similar to that for $V=0$.

The same problem occurs for the other types of impurity and in calculations of $\alpha$ from $\Delta E$, as $\Delta E(M, m)$ always approaches $\Delta E(M, \infty)$ from below. The absolute error in $\Delta E$ is of the same order of magnitude as the one in $O$.

The best way to overcome this difficulty would be an extrapolation from finite $m$ to $m=\infty$. Unfortunately the functional dependence of $O(M, m)$ and $\Delta E(M, m)$ on $m$ is not known. The numerical data for $O(M, m)$ and $\Delta E(M, m)$ show signs of a power law dependence on $1 / m$ with an exponent of the order of 4.5 , but we found it impossible to extract a convincing extrapolation law. By choosing very large $m$ we instead ensure that the systematic error is always insignificantly small. To this end we keep $m=600$ states per block for $M \in[6,48]$, $m=800$ for $M \in[50,58], m=1000$ for $M \in[62,80]$, $m=1100$ for $M \in[84,90]$, and $m=1200$ for $M=100$ for all data sets shown in the next section. For these $m$ the value of $O(M, m)-O(M, \infty)$ for $M=100$ is smaller than $10^{-6}$, and it is even less for smaller values of $M$. As we will see in Sec. IV, this level of accuracy is needed to obtain meaningful results.

As can be seen in Fig. 2 the error for the interacting data is larger than for the non-interacting ones. This observation holds for all $M, \Delta E$ and the other types of impurities.

\section{NUMERICAL RESULTS FOR INTERACTING ELECTRONS}

In this section we present and interpret numerical results for the three chosen types of local impurities. In the discussion we will distinguish between the weak impurity limit where we expect to find results given by the Eqs. (29) and (32), the weak hopping limit where we calculate the overlap with the OBC ground state and expect Eq. (34) to hold, and the strong impurity limit.

\section{A. Weak impurities}

For weak impurities and attractive interaction perturbation theory predicts that the backward scattering contribution to the overlap tends to a constant (see Eq. (32)). The remaining $M$ dependence of $O$ is given by the forward scattering contribution. To check this, and Eq. (29) for $\alpha_{f}^{B}(V)$, we calculated $O$ and $\Delta E$ for several values of $V<0$ and weak site impurities. From these data we found $\alpha^{O}[i]$ and $\alpha^{\Delta E}[i]$ as determined by Eqs. (35) and (36). Fig. 3 shows the results for the parameter sets $V /|t|=-0.75, \varepsilon /|t|=0.05, V /|t|=-1, \varepsilon /|t|=0.25$ and $V /|t|=-1.5, \varepsilon /|t|=0.1$. Assuming that at large $M$ the finite size scaling is given by integer powers of $1 / M$ Eq. (30) and not an anomalous scaling of the form Eq. (32), 


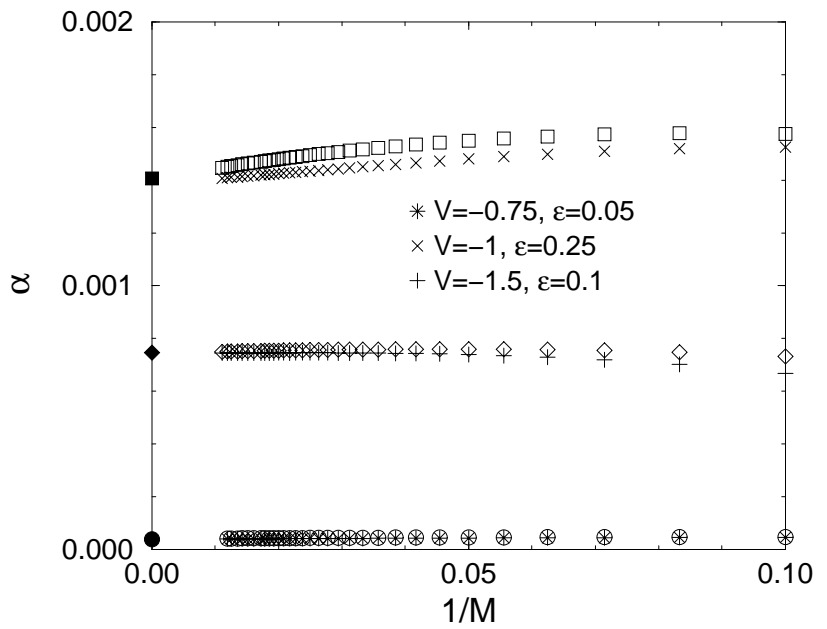

FIG. 3. The orthogonality exponent for weak site impurities and attractive interaction. The stars are $\alpha^{O}$ and the circles are $\alpha^{\Delta E}$ for $V /|t|=-0.75, \varepsilon /|t|=0.05$, the crosses are $\alpha^{O}$ and the squares are $\alpha^{\Delta E}$ for $V /|t|=-1, \varepsilon /|t|=0.25$ and the plus signs are $\alpha^{O}$ and the diamonds are $\alpha^{\Delta E}$ for $V /|t|=-1.5, \varepsilon /|t|=0.1$. The full symbols represent the related $\alpha_{f}^{B}$. In the legend $V$ and $\varepsilon$ are measured in units of $|t|$.

we can extrapolate the data by fitting polynomials up to second order in $1 / M$. As the fitting works very well this procedure is justified. In Table II we list $\alpha^{\text {exact }}(0)$, $\alpha^{B}(0), \alpha_{f}^{B}(V)$, and the extrapolated $\alpha(V)$ for a fit with $1 / M \in[0.01,0.03]$. Taking into account the extrapolation error and the fact that the Born approximation is even in the non-interacting case only an approximation (see Table II), we conclude that our data are consistent with the predictions of perturbation theory and the RG. There is no backward scattering contribution to $\alpha$ and $\alpha_{f}(V)$ is given by $\alpha_{f}^{B}(V)$. We do not believe that the difference between the analytic values of $\alpha_{f}^{B}(V)$ and the extrapolated $\alpha$ can be explained by a residual backward scattering contribution. The difference is much smaller than the backward scattering contribution for $V=0$ [as discussed following Eq. (16) $\left.\alpha_{b}(V=0)=\alpha(V=0) / 2\right]$ and for some of the parameter sets the extrapolated $\alpha$ is smaller than the analytical $\alpha_{f}^{B}(V)$. As $\alpha^{O}[i]$ and $\alpha^{\Delta E}[i]$ tend to the same limit as $M \rightarrow \infty$ the data are consistent with the results of BCFT (Eq. (10)).

We would like to emphasize that for weak impurities it is important to calculate $O$ and $\Delta E$ with a very high numerical accuracy. The difference between $O$ and 1 is very small, e. g. for $V /|t|=-0.75$ and $\varepsilon /|t|=0.05$ this difference is of the order of $10^{-4}$. Therefore results are only meaningful if $O$ and $\Delta E$ have a much smaller error. As discussed in the last section the error in $O$ and $\Delta E$ in our calculations is less than $10^{-6}$.

The next goal is to confirm Eq. (32) for weak hop-

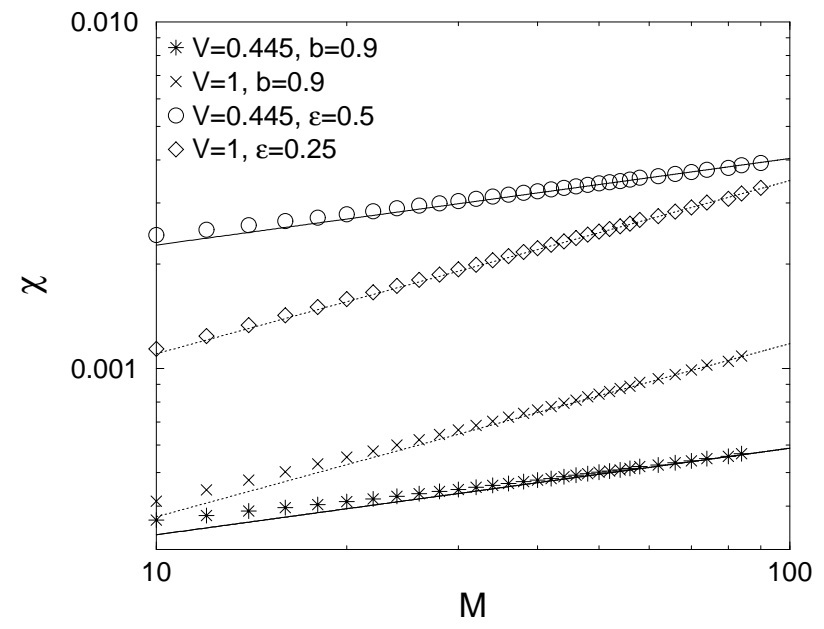

FIG. 4. Scaling behavior of the overlap for weak impurities and repulsive interaction. The symbols are the DMRG results for parameters as indicated in the legend. The solid lines are power laws with exponent $2-2 K=0.25$ and the dotted lines have the exponent $2-2 K=0.5$. In the legend $V$ and $\varepsilon$ are measured in units of $|t|$.

ping impurities, i. e. small $1-b$, with attractive and repulsive interaction and weak site impurities with repulsive interaction. In the case of the hopping impurity there is only backward scattering, and $O$ is given by $O_{b}$. For site impurities we have to distinguish between the conventional weak forward scattering contribution to $O \sim-\alpha_{f}(V) \ln (M)$ and the anomalous scaling given by Eq. (32). We have already confirmed that the analytical result Eq. (29) for $\alpha_{f}^{B}(V)$ is a good approximation for small $\varepsilon /|t|$. Therefore we can extract the anomalous behavior by subtracting $-\alpha_{f}^{B}(V) \ln (M)$. In the following $O$ always stands for the anomalous contribution.

As the constant $a_{1}(V)$ in Eq. (32) is unknown we analyze $O$ by calculating centered differences of $O$ and expect from Eq. (32)

$$
\begin{aligned}
\chi[i] & \equiv-\frac{O(M[i+1])-O(M[i-1])}{\ln \{M[i+1]\}-\ln \{M[i-1]\}} \\
& =a_{2}(V) \frac{W_{b}^{2}}{v_{F}^{2}}(2-2 K) M^{2-2 K}+\ldots .
\end{aligned}
$$

In lowest order in perturbation theory $W_{b} / v_{F}$ for the site impurity is given by $\varepsilon / v_{F}$ and for the hopping impurity by $1-b$. As discussed in the Appendix $a_{2}(V)(2-2 K) \geq 0$.

For repulsive interaction $(K<1)$ we expect $\chi$ to increase as a power law. This power law holds for $M$ with $\left(W_{b} / v_{F}\right)^{2} M^{2-2 K} \ll 1$ but $M \gg 1$. For larger $M$ the expansion Eq. (32) breaks down and for smaller $M$ the corrections symbolized by the dots become important. Fig. 4 shows a double logarithmic plot of $\chi$ as a function of $M$ for weak hopping and site impurities with different $b, \varepsilon$ 


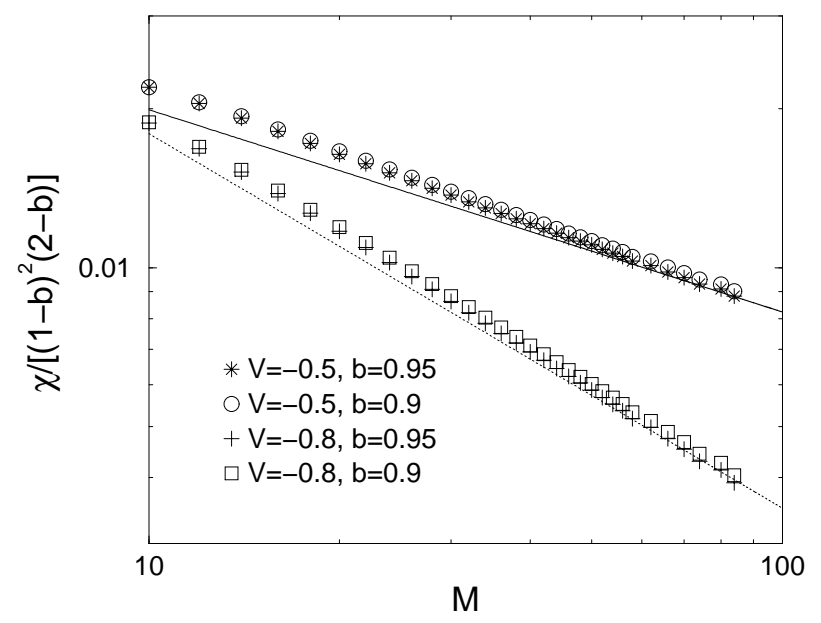

FIG. 5. Scaling behavior of the overlap for weak hopping impurities and attractive interaction. The symbols are the DMRG results for parameters as indicated in the legend. The solid line presents a power law with exponent $2-2 K=-0.3834$ and the dotted line has the exponent $2-2 K=-0.7100$. In the legend $V$ is measured in units of $|t|$.

and $V$. The lines are power laws with the expected exponent $2-2 K$. For $V=0.445$ Eq. (13) gives $2-2 K=0.25$ and for $V=1,2-2 K=0.5$. For large $M$ the DMRG results approach the predicted power law behavior. As we have chosen small $1-b$ and $\varepsilon /|t|$, the power law holds for all accessible system sizes with $M>40$.

For attractive interaction $(K>1)$ and weak hopping impurities Eq. (37) predicts that $\chi$ tends to zero as $M^{2-2 K}$. This power law behavior should hold as long as $2-2 K>-1$, i. e. for $1<K<3 / 2$. For larger $K$ the $1 / M$ correction included in the terms symbolized by the dots dominates the large $M$ behavior.

If we calculate $\chi /\left(W_{b} / v_{F}\right)^{2}$ the right hand side (rhs) of Eq. (37) is independent of $\left(W_{b} / v_{F}\right)^{2}$, i. e. we expect the data for large $M$, fixed $V$ but different $W_{b}$ to collapse onto one curve. The lowest order expression of $W_{b} / v_{F}$ in terms of the microscopic impurity parameter is given by $1-b$. We calculated $\chi /(1-b)^{2}$ for $V /|t|=-0.5$ and $V /|t|=-0.8$ each for $b=0.95$ and $b=0.9$. From Eq. (13) we obtain for $V /|t|=-0.5,2-2 K=-0.3834$ and for $V /|t|=-0.8,2-2 K=-0.7100$. Even though $1-b$ is very small the data points for the two different $b$ do not collapse onto one curve and we find a deviation between the power law behavior we expect from Eq. (37) and the behavior of the data at large $M$ (see Fig. 5). This shows that higher order corrections are of importance. From a numerical point of view it is impossible to work with smaller $1-b$, as the maximal difference between the overlap $O$ and 1 for $b=0.95$ is only $1.5 \cdot 10^{-4}$. For smaller $1-b$ this difference would be even smaller and we would get very close to the numerical accuracy of $O$.

We have to distinguish between two different sources of higher order corrections. The first of these arises in the mapping from microscopic to field theoretical impurity parameters. As discussed in the context of Eq. (20), the higher order corrections to $\alpha^{B}(V=0)$ for the hopping impurity are of the order $(1-b)^{3}$. If we include this correction and make the identification

$$
\frac{W_{b}^{2}}{v_{F}^{2}}=(1-b)^{2}[1+(1-b)]
$$

the different data sets are only weakly $b$ dependent. Even if we include these corrections the results for different $b$ do not approach one another for large $M$. Instead the difference between the $b=0.95$ and $b=0.9$ data sets slowly increases as $M$ gets larger. This can be explained by higher order corrections in Eq. (32) (see below). Fig. 5 shows a double logarithmic plot of $\chi /\left[(1-b)^{2}(2-b)\right]$ for the above parameters. We also calculated $\chi / \delta_{b}^{2}$ with the exact non-interacting backward scattering phase shift $\delta_{b}$ given by Eq. (18), but this gives no further improvement compared to the results shown in Fig. 5. We therefore conclude that even for relatively small $1-b$ it is necessary to take higher order corrections in the mapping of the microscopic impurity parameters onto the field theoretical parameters into account.

The slight deviation of the numerical data from the expected asymptotic power law behavior (shown by the lines in Fig. 5) suggests that higher order corrections are also important in the field theoretical calculation leading to Eq. (32). Unfortunately it is difficult to calculate these corrections analytically, even within the TL model. As, among the cases considered here, these effects seem to be important only for weak hopping impurities with attractive interaction, we will not pursue this question further in the present article.

We also performed DMRG calculations for weak bond impurities. The results are equivalent to the results we obtained for hopping impurities.

In summary, we conclude that our highly accurate DMRG data show the expected weak impurity behavior in most of the cases discussed. For site impurities and attractive interaction we have numerically verified the Eq. (29) for $\alpha_{f}^{B}(V)$. The backward scattering contribution to $\alpha$ does vanish. For weak site, hopping, and bond impurities with repulsive interaction the data are in agreement with the scaling law Eq. (32). Only for weak hopping and bond impurities and attractive interaction we found small deviations between the scaling law Eq. (32) and the numerical results. In this case higher order corrections are of importance.

\section{B. Weak hopping in an open chain}

To verify Eq. (34) for our microscopic lattice model we calculated the overlap $O^{\circ}$ between the chains with $\mathrm{OBC}$ 


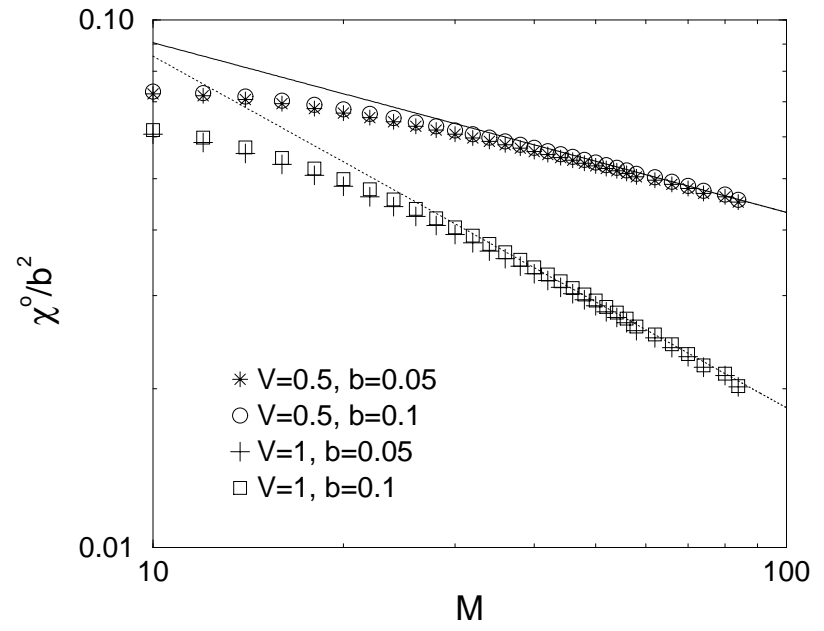

FIG. 6. Scaling behavior of the overlap $O^{\circ}$ between the open chain and a chain with a weak hopping for repulsive interaction. The symbols are the DMRG results for parameters as indicated in the legend. The solid line is a power law with the expected exponent $2-2 / K=-0.3217$ and the dotted line has exponent $2-2 / K=-2 / 3$. In the legend $V$ is measured in units of $|t|$.

(hopping impurity with $b=0$ ) and with a strong hopping impurity, i. e. small $t_{b}$, for repulsive interaction. There is no forward scattering contribution to the overlap and $O^{o}$ is given by $O_{b}^{o}$ alone. Again it is very important to have numerical data for $O^{\circ}$ with a large accuracy as the difference between $O^{\circ}$ and 1 is of the order of $10^{-4}$.

The constant $\tilde{a}_{1}(V)$ in Eq. (34) is unknown and as above we analyze $O^{\circ}$ by calculating centered differences. For these we expect

$$
\begin{aligned}
\chi^{o}[i] & \equiv-\frac{O^{o}(M[i+1])-O^{o}(M[i-1])}{\ln \{M[i+1]\}-\ln \{M[i-1]\}} \\
& =\tilde{a}_{2}(V) \frac{t_{b}^{2}}{t^{2}}(2-2 / K) M^{2-2 / K}+\ldots,
\end{aligned}
$$

to hold. As in the weak impurity case we have $\tilde{a}_{2}(V)(2-$ $2 / K) \geq 0$. Provided that $2-2 / K>-1$ and $K<1$ (i. e. $2 / 3<K<1$ ) $\chi^{o}[i]$ decays as a power law in $1 / M$, with exponent $2-2 / K$. For smaller $K$ the $1 / M$ correction to Eq. (39) (among the terms indicated by the dots), dies away more slowly than the $M^{2-2 / K}$ term, and dominates the behavior at large $M$. Furthermore if we divide both sides of Eq. (39) by $\left(t_{b} / t\right)^{2}$ the rhs is independent of $t_{b}$. For the weak hopping case $\left(t_{b} / t\right)^{2}$ is given by $b^{2}$. Therefore Eq. (39) predicts that the data for large $M$, different $b$, but the same $V$ collapse onto the same curve. Fig. 6 shows a plot of $\chi^{\circ} / b^{2}$ as a function of $M$ for $V /|t|=0.5$ and $V /|t|=1$, each for $b=0.05$ and $b=0.1$. The analytical exponents $2-2 / K$ for these $V$ are -0.3217 and $-2 / 3$. The lines are power laws with the expected exponents.

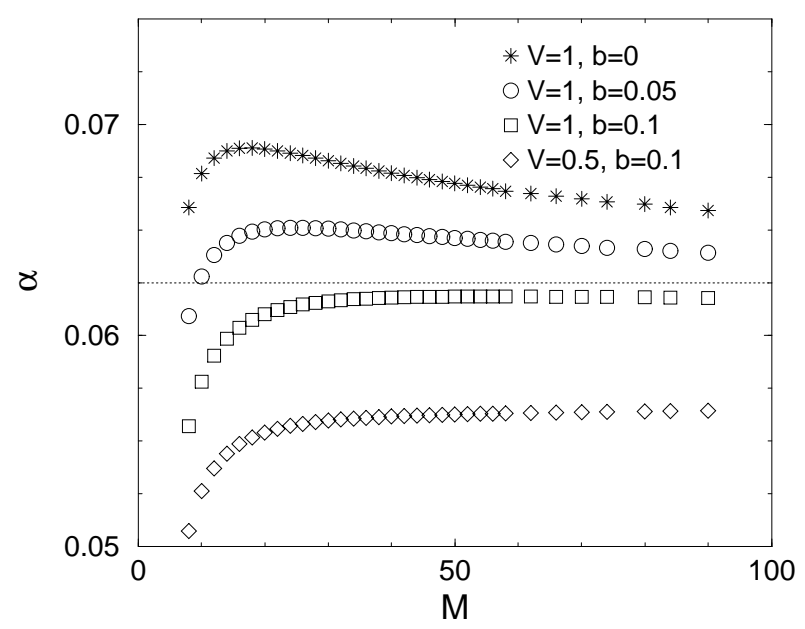

FIG. 7. Behavior of $\alpha^{O}[i]$ for a repulsive interaction in the limit of strong bond impurity. The symbols are the DMRG results for parameters as indicated in the legend. The dotted line is $\alpha=1 / 16$. In the legend $V$ is measured in units of $|t|$.

As predicted by perturbation theory, the data for different $b$ but the same $V$ asymptotically collapse onto one curve. For large $M$ these curves show power law behavior with the expected exponent $2-2 / K$. The numerical results are consistent with Eq. (34), i. e. the $\mathrm{OE} \alpha^{o}$ tends to zero and the $\mathrm{OBC}$ ground state and the ground state with a weak hopping are contrary to the non-interacting case not orthogonal to each other.

\section{Strong impurities}

The main question that we will address in this section is whether the backward scattering contribution to the OE for repulsive interaction tends to $1 / 16$ as $M \rightarrow \infty$, i. e. the open chain interpretation is valid. To avoid complications due to the forward scattering contribution to $\alpha$ we concentrate on the hopping and bond impurities. Results for strong site impurities will be discussed elsewhere [39].

In Ref. [30] Qin et al. also discuss the question of whether $\alpha_{b}$ tends to $1 / 16$ for a bond impurity with $b=0.1, V /|t|=1$ and up to 48 sites by plotting $\alpha^{O}[i]$ (see Eq. (35)). As described in Sec. III A the extrapolation error in $\alpha^{O}[i]$ for a bond impurity is much larger than that for a site impurity, i. e. the finite size corrections are much more important. Because of the anomalous corrections expected for interacting electrons (see Eq. (32)) we cannot extrapolate by fitting a polynomial in $1 / M$. It is therefore questionable whether we can extract the $M \rightarrow \infty$ limit by plotting the bare data for $\alpha^{O}[i]$.

Fig. 7 shows $\alpha^{O}[i]$ for several $V$ and $b$. The squares 
indicate data calculated using the same parameters as used in Ref. [30], but for much larger system sizes. Provided that the open chain interpretation of the $R G$ and perturbative arguments is correct, all the curves should converge to $1 / 16$ in the limit $M \rightarrow \infty$. Contrary to Qin et al. we find that it is impossible to confirm or refute this prediction by plotting $\alpha^{O}[i]$. Within the range of system sizes accessible, none of the curves saturates at $1 / 16$ finite $M$ corrections dominate the scaling for all values of b. We note that the errors of the data presented in Fig. 7 are in all cases smaller than the symbol size. It is known from BCFT that $\alpha^{O}[i]$ approaches $1 / 16$ for $b=0$ [24], but even this simple case is not obviously confirmed by Fig. 7. The behavior of the data for the hopping impurity is similar.

We have tested various different kinds of extrapolation procedure (e. g. second order polynomials and polynomials combined with terms which include anomalous scaling) as fits to the behavior of $\alpha^{O}[i]$ in the limit $M[i] \rightarrow \infty$, but none of these proved satisfactory for either the bond or the hopping impurities. For $\alpha^{O}[i]$ the corrections to the $M \rightarrow \infty$ result are too large. In the following we therefore concentrate on $\Delta E$. This means that we from now on assume that we can use the BCFT result Eq. (10) to calculate the $\mathrm{OE} \alpha$. In addition we will continue the analysis focusing on the more physical hopping impurity.

At the RG fixed point $b=b^{*}=0$ the finite $M$ corrections to $\Delta E$ are given by integer powers of $1 / M[24,31]$

$$
\begin{aligned}
\Delta E\left(V, b^{*}\right)= & c_{0}+c_{1}\left(V, b^{*}\right) M^{-1} \\
& +c_{2}\left(V, b^{*}\right) M^{-2}+\ldots,
\end{aligned}
$$

with constants $c_{i}$, and the quadratic extrapolation of $\alpha^{\Delta E}[i]$ for $M[i] \rightarrow \infty$ can be applied (see Eq. (36)). We extrapolated $\alpha^{\Delta E}[i]$ for the hopping impurity as in the Figs. 1 and 3 and obtained $\alpha_{\text {extr }}^{\Delta E}(V /|t|=0.5)=$ $0.06252, \alpha_{\text {extr }}^{\Delta E}(V /|t|=1)=0.06261$, and $\alpha_{\text {extr }}^{\Delta E}(V /|t|=$ $1.5)=0.06285$. The absolute extrapolation error for non-interacting electrons and a strong hopping impurity (discussed in Sec. III A) is of the order of $10^{-5}$ (see Table I). The extrapolated $\alpha$ for $V /|t|=0.5$ is consistent with the value $\alpha=1 / 16$, within this error. For $V /|t|=1$ and $V /|t|=1.5$ the difference between the extrapolated $\alpha$ and $1 / 16$ is slightly larger. Taking into account the error due to the approximate nature of the DMRG and the fact that the extrapolation error might be larger for interacting electrons, we can still conclude that the results for hopping impurities with $b=0$ are consistent with $\alpha=1 / 16$. The same holds for bond impurities.

For $b \neq 0$ we expect anomalous corrections in the finite size behavior of $\Delta E(V, b)$ and therefore to $\alpha^{\Delta E}[i]$. Unfortunately we cannot extract the anomalous scaling behavior directly from our data for $\alpha^{\Delta E}[i]$, as it is masked by the normal behavior Eq. (40). From the RG flow equations given in Ref. [10], we expect that close to the $b^{*}=0$ fixed point the effective $b$ is given by $b M^{-\eta}$, with $-\eta=1-1 / K$, i. e. $b$ flows to zero as we increase the system size. The idea is now to replace $b^{*}$ in Eq. (40) by $b M^{-\eta}$ and expand the difference between $\Delta E(V, b)$ and $\Delta E\left(V, b^{*}\right)$ in $b M^{-\eta}$. Before doing this it is important to notice that the constant $c_{0}$ in Eq. (40) is a functional of the Fourier transform of the impurity potential $\tilde{W}_{b}(k)$ and not only a function of the effective backward scattering strength. Therefore we cannot introduce the effective $b$ in this term and the leading term in the difference is an unknown constant $\tilde{c}_{0}$. The expansion gives

$$
\begin{aligned}
& \Delta E(V, b)-\Delta E\left(V, b^{*}\right)=\tilde{c}_{0}+\left.b \frac{\partial c_{1}(V, b)}{\partial b}\right|_{b=b^{*}} M^{-1-\eta} \\
& +\left.b \frac{\partial c_{2}(V, b)}{\partial b}\right|_{b=b^{*}} M^{-2-\eta}+\ldots \\
& +\left.\frac{1}{2} b^{2} \frac{\partial^{2} c_{1}(V, b)}{\partial b^{2}}\right|_{b=b^{*}} M^{-1-2 \eta}+\ldots
\end{aligned}
$$

Taking the derivative (numerically the centered differences) of this expression with respect to $1 / M$ we arrive at

$$
\begin{aligned}
& \Delta_{E}(V, b) \equiv \frac{\partial\left\{\Delta E(V, b)-\Delta E\left(V, b^{*}\right)\right\}}{\partial(1 / M)} \\
& =\left.b \frac{\partial c_{1}(V, b)}{\partial b}\right|_{b=b^{*}}(1+\eta) M^{1-1 / K} \\
& +\left.b \frac{\partial c_{2}(V, b)}{\partial b}\right|_{b=b^{*}}(2+\eta) M^{-1 / K}+\ldots \\
& +\left.\frac{1}{2} b^{2} \frac{\partial^{2} c_{1}(V, b)}{\partial b^{2}}\right|_{b=b^{*}}(1+2 \eta) M^{2-2 / K}+\ldots .
\end{aligned}
$$

Provided we can use the scaling law for the effective $b$ in the derivation of Eq. (41) we expect that our data can be described by Eq. (42).

Fig. 8 shows $-\Delta_{E} /(b|t|)$ as a function of $M$ for a hopping impurity with $V /|t|=0.5, V /|t|=1$ and $V /|t|=1.5$, for $b=0.025, b=0.05$ and $b=0.1$ (for a discussion of the data for $b=0.3$ and $b=0.4$ see below). The data for the different $V$ and large $M$ collapse onto a single curve. As we expect from Eq. (42), the $b$ dependent corrections [e. g. the term in the fourth line of Eq. (42)] are more important for weak interaction, i. e. $K$ closer to 1 , than for strong interaction. It is obvious from Fig. 8 that, for the accessible system sizes, the numerical data cannot be described by the leading term of Eq. (42) alone. Taking only the powers of $M$ into account the term proportional to $b^{2}$ in Eq. (42) seems to be a more important finite size correction than that proportional to $b M^{-1 / K}$. But as the data for small $b$ and larger $V$ have already collapsed onto a single curve for $M>50$, we conclude that the term proportional to $b^{2}$ is already small and neglect this contribution in what follows. To verify that the data fall away as predicted we fitted the points for $b=0.025$ and $M \geq 30$ with a function of the form

$$
f(M)=d_{1} M^{1-1 / \tilde{K}}+d_{2} M^{-1 / \tilde{K}},
$$

with fitting parameters $d_{1}, d_{2}$, and $\tilde{K}$. The fits are shown as lines in Fig. 8. The exact values for $K$ are $K=0.861$, 


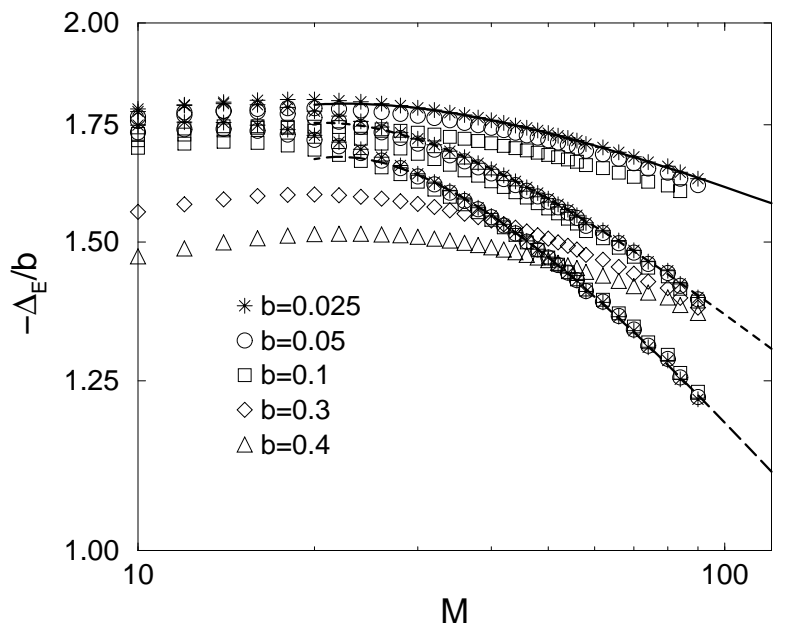

FIG. 8. Large $M$ behavior of $-\Delta_{E} /(b|t|)$ for repulsive interaction and a strong hopping impurity. For each interaction strength $V /|t|=0.5, V /|t|=1$, and $V /|t|=1.5$ the figure shows data for $b=0.025, b=0.05$, and $b=0.1$. The top set of curves is for $V /|t|=0.5$, the set in the middle for $V /|t|=1$, and the bottom set for $V /|t|=1.5$. The lines are fits to the data (for details see the text). The diamonds and triangles are data for $b=0.3$ and $b=0.4$ both for $V /|t|=1$. In the figure $\Delta_{E}$ is measured in units of $|t|$.

for $V /|t|=0.5, K=3 / 4$, for $V /|t|=1$, and $K=0.649$, for $V /|t|=1.5$. These numbers must be compared with the exponents $\tilde{K}=0.873, \tilde{K}=0.774$, and $\tilde{K}=0.706$ obtained by fitting the data. For $V /|t|=0.5$ and $V /|t|=1$ the agreement between the fitted and the expected $K$ is satisfactory. For large interaction the agreement is not that good. In this case higher order corrections proportional to $b$ seem to be more important. If we fit the data in a smaller $M$ window, i. e. only for the few largest system sizes available, the difference between the fitted and exact $K$ is decreasing as it should be.

We conclude that for $M \rightarrow \infty$ the $M$ dependent part of $\Delta E(V, b)$ approaches the $M$ dependent part of the fixed point energy difference $\Delta E\left(V, b^{*}=0\right)$ in the way expected from RG. In particular we find no sign of a term of order $M^{0}$ in $\Delta_{E}(V, b)$. If $\alpha^{\Delta E}(V, b)$ would be different from $\alpha^{\Delta E}\left(V, b^{*}\right), \Delta_{E}(V, b)$ would contain such a constant given by $2 \pi v\left[\alpha^{\Delta E}(V, b)-\alpha^{\Delta E}\left(V, b^{*}\right)\right]$. By plotting $\Delta_{E}(V, b)$ as in Fig. 8 we are able to detect such a constant if it is up to 100 times smaller than $2 \pi v_{F}\left[\alpha^{\Delta E}(V=0, b)-\alpha^{\Delta E}\left(V=0, b^{*}\right)\right]$. As the numerical results for $\alpha_{\text {extr }}^{\Delta E}\left(V, b^{*}\right)$ are consistent with $\alpha=1 / 16$ it follows that $\alpha=1 / 16$ holds also for small $b$ away from the fixed point value provided that the use of BCFT predictions is valid. We wish to emphasize that we can give quantitative numerical evidence for the predicted RG flow of the ground state energy difference for a fixed $b$, and not only a qualitative picture of the flow by choosing different $b$ as was obtained in Ref. [31].

We performed the same kind of analysis for the bond impurity. The results are similar to those presented for the more physical hopping impurity and the same conclusions can be drawn.

High accuracy DMRG then provides indirect numerical evidence that, for repulsive interaction, the backward scattering contribution to the OE for the overlap between the PBC ground state and the state with a local impurity is independent of both interaction and impurity strength, and has the universal value $1 / 16$. This result is consistent with those obtained by approximative analytical calculations in the effective low energy field theory based on the open chain interpretation and discussed in the introduction.

Even though we have studied chains with up to 100 sites, very small $b$ and obtain DMRG data with a very high accuracy, we can only give indirect evidence for this universal behavior because finite size corrections are very large. To show directly that $\alpha^{O}[i]$ (and $\alpha^{\Delta E}[i]$ ) tend to $1 / 16$ would require much larger system sizes.

Fig. 8 also contains data for $b=0.3, b=0.4$ and $V /|t|=1$. For the accessible system sizes the data points for these larger values of $b$ are further away from the asymptotic curve onto which the data for smaller $b$ collapse. However the points do seem to converge with this curve for large $M$. One can therefore speculate that the backward scattering contribution to the $\mathrm{OE}$ is also $1 / 16$ for larger values of $b$. This is consistent with the open chain interpretation of the $R G$ results.

\section{SUMMARY}

In this article we presented the results of an extremely accurate DMRG study of the orthogonality catastrophe in a 1D lattice model of correlated electrons. We considered three different types of impurity and a range of values of the interaction. By making a detailed comparison with exact results for non-interacting electrons we were able to establish the accuracy of our DMRG data, and arrive at a method of extrapolating our finite size results to the thermodynamic limit.

For weak impurities we found that, in most of the cases considered, the numerical data can be well described by the results of a analytical calculation in lowest order perturbation theory in the impurity strength. The perturbation theory is performed within the effective low energy continuum field theory for 1D correlated electrons. Higher order corrections are important in the case of weak hopping and bond impurities with attractive interaction. We have confirmed numerically that a weak backward scattering component to the OE scales to zero for attractive interaction and that the forward scattering contribution is modified by the interaction. The data of the overlap also show an interaction dependent anomalous scaling with system size which was predicted by per- 
turbation theory in the (backward scattering) impurity strength.

In the case of a strong impurity we were able to confirm indirectly the RG prediction that the backward scattering contribution to the $\mathrm{OE}$ tends to $1 / 16$ in the thermodynamic limit. This value is universal and independent of both impurity strength and the size of the interaction. Contrary to the conclusion drawn in previous studies by Qin et al. we found that direct evidence for this behavior cannot be extracted from data for the overlap at the accessible system sizes, because the finite size corrections are very large. However we obtained quantitative numerical evidence for the scaling of the ground state energy difference predicted by RG.

The open chain interpretation of perturbative RG results leads to the prediction that $\alpha_{b}(V>0)$ scales to $1 / 16$ in the presence of an impurity with an arbitrarily weak backward scattering component (e. g. a hopping or bond impurity with $b \geq 0.9)$. By close analogy $\alpha_{b}(V<0)$ should then tend to 0 even for very strong bare backward scattering (e. g. a hopping or bound impurity with $b \leq 0.1$ ). We found it impossible to confirm these predictions quantitatively on the basis of DMRG data for systems with up to 100 sites. The finite size corrections are in all cases too large, and the scaling too slow. Much larger systems are needed to make a reliable numerical determination of these issues possible. The results are however in qualitative agreement with this extension of the $\mathrm{RG}$ results.

\section{ACKNOWLEDGMENTS}

We would like to thank the ISI Foundation for making possible the workshop on "The Role of Dimensionality in Correlated Electronic Systems" at Villa Gualino (Torino, Italy) in May 1996 where this work was begun. We are also grateful to the EU (HC\&M Network ERBCHRX-CT920020) for financial support during our stay in Torino. Most of the numerical calculations were done on the IBM SP2 cluster at Indiana University. We would like to thank the University Computing Services for support. Additional calculations were done on the IBM SP2 at the Leibniz-Rechenzentrum in Munich. We are grateful to C. Schuster for useful comments on the manuscript.

V. M. would like to thank K. Schönhammer, S. Girvin, W. Zwerger, S. Eggert, W. Atkinson, and U. Zülicke for discussions and the Deutsche Forschungsgemeinschaft and the NSF Grant No. DMR-9416906 for financial support. This work was partially supported through the TMR program of the European Union (P. S.). N. S. would like to acknowledge useful conversations with $\mathrm{F}$. Eßler, B. Muzikanski, and N. d'Ambrumenil, and support under an EPSRC grant.

\section{APPENDIX}

In this Appendix we will briefly describe how to obtain the perturbative expressions Eqs. (32) and (34) for the overlap [6]. By $\mathcal{H}_{0}$ we denote a general Hamiltonian and by $\mathcal{W}$ the perturbation. The overlap between the ground state $\left|E_{0}\right\rangle$ of $\mathcal{H}_{0}$ and $\left|E_{0}^{I}\right\rangle$ of $\mathcal{H}_{0}+\mathcal{W}$ is given by

$$
O^{2} \equiv\left|\left\langle E_{0} \mid E_{0}^{I}\right\rangle\right|^{2}=1-\sum_{n \neq 0}\left|\left\langle E_{0} \mid E_{n}^{I}\right\rangle\right|^{2}
$$

where $\left|E_{n}^{I}\right\rangle$ are the excited eigenstates of $\mathcal{H}_{0}+\mathcal{W}$. Inserting the expression for $\left|E_{n}^{I}\right\rangle$ in lowest order perturbation theory in $\mathcal{W}$ gives

$$
O^{2} \approx 1-\sum_{n \neq 0} \frac{\left|\left\langle E_{0}|\mathcal{W}| E_{n}\right\rangle\right|^{2}}{\left(E_{n}-E_{0}\right)^{2}}
$$

By introducing a $\delta$ function and taking into account that the smallest energy difference $E_{n}-E_{0}$ is proportional to $1 / L$ we end up with

$$
O^{2} \approx 1-\int_{2 \pi / L}^{\infty} \frac{d \nu}{\nu^{2}}\left\langle E_{0}\left|\mathcal{W} \delta\left(\nu-\left[\mathcal{H}_{0}-E_{0}\right]\right) \mathcal{W}\right| E_{0}\right\rangle
$$

We will now specify $\mathcal{H}_{0}$ and $\mathcal{W}$ to be given by the Eqs. (21) and (31). In this case the correlation function occurring in Eq. (46) is related to the $2 k_{F}$ component of the density-density correlation function in the TL model. For the TL Hamiltonian it is known how to calculate correlation functions like the one occurring in Eq. (46) [23]. Evaluating the expression gives

$$
\begin{aligned}
O_{b}^{2} \approx & 1-c_{1}(V) \frac{W_{b}^{2}}{v_{F}^{2}} \\
& -c_{2}(V) \frac{2}{4 \pi^{2}} \frac{W_{b}^{2}}{v_{F}^{2}} \frac{L^{2-2 K}-1}{2-2 K}
\end{aligned}
$$

with positive constants $c_{i}$. An explicit expression for $c_{2}(V)$ can be given, but in the following we only need to know that $c_{2}(V=0)=1[12]$. Eq. (47) is equivalent to Eq. (32). In the limit $K \rightarrow 1$, i. e. $V \rightarrow 0$, $\left(L^{2-2 K}-1\right) /(2-2 K)$ tends to $\ln (L)$ and we recover Eq. (33).

Taking the dual Hamiltonian describing an open chain and a representation of the weak hopping in terms of the dual field theory [10] instead of Eqs. (21) and (31) we can perform a similar kind of calculation and end up with Eq. (34).

In the non-interacting case we can furthermore write down higher order corrections. They sum up to the expected $O_{b} \sim \exp \left\{-\alpha_{b} \ln (L)\right\}$ behavior and finite size corrections that are integer powers of $1 / L$. 
[1] P. W. Anderson, Phys. Rev. Lett. 18, 1049 (1967).

[2] P. Noziéres and C. T. de Dominicis, Phys. Rev. 178, 1097 (1969).

[3] G. D. Mahan, Many-Particle Physics, (Plenum Press, New York 1981).

[4] K. Yamada and K. Yoshida, Prog. Theor. Phys. 68, 1504 (1982).

[5] D. R. Hamann, Phys. Rev. Lett. 26, 1030 (1971).

[6] K. Schönhammer, Phys. Rev. B 43, 11323 (1991).

[7] F. D. M. Haldane, J. Phys. C 14, 2585 (1981).

[8] S. R. White, Phys. Rev. Lett. 69, 2863 (1992); S. R. White and R. M. Noak, Phys. Rev. Lett. 68, 3487 (1992); S. R. White, Phys. Rev. B 48, 10245 (1993).

[9] for the adaptation of the algorithm in the presence of impurities see also P. Schmitteckert, PhD-Thesis, Univeristät Augsburg (1996), http://www.physik.uniaugsburg.de/ peters/thesis.

[10] C. L. Kane and M. P. A. Fisher, Phys. Rev. B 46, 15233 (1992).

[11] K. Moon, C. L. Kane, S. M. Girvin, and M. P. A. Fisher, Phys. Rev. Lett. 71, 4381 (1993).

[12] V. Meden, Diploma-Thesis, Universität Göttingen (1992).

[13] R. Egger and H. Grabert, Phys. Rev. Lett. 75, 3505 (1995).

[14] P. Schmitteckert and U. Eckern, Phys. Rev. B 53, 15397 (1996).

[15] P. Fendley, A. W. W. Ludwig, and H. Saleur, Phys. Rev. B 52, 8934 (1995).

[16] F. Lesage, H. Saleur, and P. Simonetti, preprint, condmat/9703220 (1997).

[17] A. O. Gogolin, Phys. Rev. Lett. 71, 2995 (1993); JETP Lett. 57, 50 (1993).

[18] N. V. Prokof'ev, Phys. Rev. B 49, 2148 (1994).

[19] C. L. Kane, K. A. Matveev, and L. I. Glazman, Phys. Rev. B 49, 2253 (1994).

[20] Y. Oreg and A. M. Finkel'stein, Phys. Rev. Lett. 76, 4230 (1996).

[21] Y. Oreg and A. M. Finkel'stein, Phys. Rev. B 53, 10928 (1996).

[22] M. Fabrizio and A. O. Gogolin, Phys. Rev. Lett. 78, 4527 (1997); Y. Oreg and A. M. Finkel'stein, Phys. Rev. Lett. 78, 4528 (1997).

[23] see e. g. J. Voit, Rep. Prog. Phys. 58, 977 (1995).

[24] I. Affleck and A. W. W. Ludwig, J. Phys. A: Math. Gen. 27, 5375 (1994).

[25] A. M. Zagoskin and I. Affleck, preprint, cond-mat/9704248 (1997).

[26] for the isotropic Heisenberg model see also S. Eggert and I. Affleck, Phys. Rev. B 46, 10866 (1992).

[27] F. H. L. Eßler and H. Frahm, preprint, cond-mat/9702234 (1997).

[28] A. Furusaki, preprint, cond-mat/9702195 (1997).

[29] A. Komnik, R. Egger, and A. O. Gogolin, preprint, condmat/9702238 (1997).

[30] S. Qin, M. Fabrizio, and L. Yu, Phys. Rev. B 54, R9643 (1996).

[31] S. Qin, M. Fabrizio, L. Yu, M. Oshikawa, and I. Affleck, preprint, cond-mat/9705269 (1997).

[32] F. Woynarovich and H. P. Eckle, J. Phys. A 20, L97
(1987); C. J. Hamer, G. R. W. Quispel, and M. T. Batchelor, J. Phys. A: Math. Gen. 20, 5677 (1987).

[33] H. Bethe, Z. Phys. B 71, 205 (1931); C. N. Yang and C. P. Yang, Phys. Rev. 150, 321 (1966); ibid., 327 (1966).

[34] P. Jordan and E. Wigner, Z. Phys. 47, 631 (1928); E. Lieb, T. Schultz, and D. Mattis, Annals of Physics 16, 407 (1961).

[35] K. Schönhammer, Prog. Theor. Phys. Supplement 106, 147 (1991).

[36] T. Ogawa, A. Furusaki, and N. Nagaosa, Phys. Rev. Lett. 68, 3638 (1992); D. K. K. Lee and Y. Chen, Phys. Rev. Lett. 69, 1399 (1992).

[37] K. D. Schotte and U. Schotte, Phys. Rev. 182, 479 (1969).

[38] M. Abramowitz and I. A. Stegun, Handbook of Mathematical Functions, (Dover, New York, 1970).

[39] V. Meden, P. Schmitteckert, and N. Shannon, in preparation. 


\begin{tabular}{lcccc}
\hline \hline & exact & error from $O$ & error from $\Delta E$ & error from $O$ \\
& $M \in[200,1200]$ & $M \in[200,1200]$ & $M \in[50,200]$ & error from $\Delta E$ \\
site & $4.89322299 \cdot 10^{-2}$ & $1 \cdot 10^{-7}$ & $1 \cdot 10^{-9}$ & $2 \cdot 10^{-5}$ \\
hopping & $4.76437497 \cdot 10^{-2}$ & $5 \cdot 10^{-5}$ & $3 \cdot 10^{-7}$ & $2 \cdot 10^{-4}$ \\
\hline \hline
\end{tabular}

TABLE I. The first column gives the exact value of the OE $\alpha$ in the non-interacting tight binding Hamiltonian for a site impurity with $\varepsilon /|t|=3$ and a hopping impurity with $b=0.1$. The other columns are the errors obtained by extrapolating the data of Fig. 1. The second and third columns are the errors for an extrapolation with $M \in[200,1200]$ and the fourth and fifth the ones for $M \in[50,200]$.

\begin{tabular}{ccccc}
\hline \hline & $\alpha^{\text {exact }}(0)$ & $\alpha^{B}(0)$ & $\alpha_{f}^{B}(V)$ & $\alpha_{\text {extr }}^{O}(V)$ \\
$V=-0.75, \varepsilon=0.05$ & $3.1650 \cdot 10^{-5}$ & $3.1663 \cdot 10^{-5}$ & $3.7789 \cdot 10^{-5}$ & $3.8152 \cdot 10^{-5}$ \\
$V=-1, \varepsilon=0.25$ & $7.8342 \cdot 10^{-4}$ & $7.9157 \cdot 10^{-4}$ & $1.4072 \cdot 10^{-3}$ & $1.3862 \cdot 10^{-3}$ \\
$V=-1.5, \varepsilon=0.1$ & $1.2644 \cdot 10^{-4}$ & $1.2665 \cdot 10^{-4}$ & $7.4598 \cdot 10^{-4}$ & $7.4128 \cdot 10^{-4}$ \\
\hline \hline
\end{tabular}

TABLE II. The OE for weak site impurities. The first column gives the exact non-interacting $\alpha$, the second column the non-interacting Born approximation, the third column the forward scattering contribution of the interacting Born approximation, and the fourth and fifth columns the extrapolated values from Fig. 3. The extrapolation is done by a quadratic fit with $1 / M \in[0.01,0.03]$. In the Table $V$ and $\varepsilon$ are measured in units of $|t|$. 